\title{
Spin dynamics in cuprate perovskites
}

\author{
A. Sherman ${ }^{1}$ and M. Schreiber ${ }^{2}$ \\ 1 Institute of Physics, University of Tartu, Riia 142, Tartu, 51014 Estonia \\ E-mail: alexei@fi.tartu.ee \\ ${ }^{2}$ Institut für Physik, Technische Universität, D-09107 Chemnitz, Germany \\ E-mail: schreiber@physik.tu-chemnitz.de
}

Received June 8, 2005

\begin{abstract}
Results obtained with the use of the $t-J$ model of $\mathrm{Cu}-\mathrm{O}$ planes and Mori's projection operator formalism are compared with data of neutron scattering experiments in lanthanum and yttrium cuprates. This comparison allows us to interpret the intensive peak at the antiferromagnetic wave vector observed in yttrium cuprates as a manifestation of excitations of localized $\mathrm{Cu}$ spins. The high-frequency incommensurability detected both in lanthanum and yttrium cuprates is connected with the dispersion of these excitations, while the low-frequency incommensurability arises due to a dip in the spin-excitation damping at the antiferromagnetic wave vector. For moderate doping the dip stems from the weakness of the interaction between the spin excitations and holes near hot spots. It is conjectured that the dissimilarity of the susceptibility frequency dependencies in yttrium and lanthanum cuprates may be connected with different values of the hole bandwidth and damping in these crystals.
\end{abstract}

PACS: 71.10.Fd, 74.25.Ha, 74.72.-h

Keywords: spin dynamics, dispersion of spin excitation.

One of the most interesting features of the inelastic neutron scattering in lanthanum cuprates is that for hole concentrations $x \geq 0.04$, low temperatures, and small energy transfers the scattering is peaked at incommensurate momenta $(1 / 2,1 / 2 \pm \delta),(1 / 2 \pm \delta, 1 / 2)$ in the reciprocal lattice units $2 \pi / a$ with the lattice period $a$ [1]. For $x \leq 0.12$ the incommensurability parameter $\delta$ is approximately equal to $x$ and saturates for larger concentrations [2]. The incommensurability was observed both below and above $T_{c}$ [3]. The analogous low-frequency incommensurability was observed also in $\mathrm{YBa}_{2} \mathrm{Cu}_{3} \mathrm{O}_{7-y}$ [4]. This gives ground to suppose that the incommensurability is a common feature of cuprate perovskites. However, for larger frequencies the susceptibility differs essentially in these two types of cuprates. In the underdoped $\mathrm{YBa}_{2} \mathrm{Cu}_{3} \mathrm{O}_{7-y}$ and some other cuprates both below and above $T_{c}$ a pronounced maximum is observed at frequencies $\omega_{r} \approx$ $\approx 25-40 \mathrm{meV}$ [5]. In the momentum space the magnetic response is sharply peaked at the antiferromagnetic wave vector $\mathbf{Q}=(1 / 2,1 / 2)$ for this frequency. Contrastingly, no maximum at such frequencies was observed in lanthanum cuprates. Instead for low temperatures and frequencies of several millielectronvolts a broad feature was detected [6]. For even larger frequencies the magnetic response becomes again incommensurate in both types of cuprates with maxima located at momenta $(1 / 2 \pm \delta, 1 / 2 \pm \delta)$, $(1 / 2 \pm \delta, 1 / 2 \mp \delta)$ in some experiments [5,7-9]. In contrast to the low-frequency incommensurability in which the incommensurability parameter decreases with increasing frequency, the parameter of the highfrequency incommensurability grows with frequency. Thus, the dispersion of the maxima in the susceptibility resembles two parabolas with upward- and downward-directed branches which converge at the momentum $\mathbf{Q}$ and near the frequency $\omega_{r}[4,9]$.

The nature of the magnetic incommensurability is the subject of active discussion now. The most frequently used approaches for its explanation are based on the picture of itinerant electrons with the susceptibility calculated in the random phase approximation $[10,11]$ and on the stripe picture $[9,12]$.

In the present work we use an alternative approach for the interpretation of the incommensurability. In contrast to the stripe picture we do not postulate the 
existence of stripes from the outset, supposing that the magnetic incommensurability is the cause rather than the effect of stripes. Contrary to the itinerant-electron picture we take proper account of strong electron correlations inherent in underdoped cuprates by using the $t-J$ model of $\mathrm{Cu}-\mathrm{O}$ planes and the general formula for the magnetic susceptibility derived in Mori's projection operator formalism [13]. In this approach the mentioned peculiarities of the magnetic properties of cuprates in the normal state are reproduced including the proper frequency and momentum location of the susceptibility maxima.

The Hamiltonian of the two-dimensional $t-J$ model reads [14]

$$
h=\sum_{\mathbf{n m} \sigma} t_{\mathbf{n m}} a_{\mathbf{n} \sigma}^{\dagger} a_{\mathbf{m} \sigma}+\frac{1}{2} \sum_{\mathbf{n m}} J_{\mathrm{nm}} \mathbf{s}_{\mathbf{n}} \mathbf{s}_{\mathbf{m}},
$$

where $a_{\mathbf{n} \sigma}=|\mathbf{n} \sigma\rangle\langle\mathbf{n} 0|$ is the hole annihilation operator, $\mathbf{n}$ and $\mathbf{m}$ label sites of the square lattice formed by $\mathrm{Cu}$ ions, $\sigma= \pm 1$ is the spin projection, $J_{\mathrm{nm}}$ and $t_{\mathrm{nm}}$ are the exchange and hopping constants, respectively, $|\mathbf{n} \sigma\rangle$ and $|\mathbf{n} 0\rangle$ are states corresponding to the absence and presence of a hole on the site, the spin$1 / 2$ operators can be written as

$$
s_{\mathbf{n}}^{z}=\frac{1}{2} \sum_{\sigma} \sigma|\mathbf{n} \sigma\rangle\langle\mathbf{n} \sigma| \text { and } s_{\mathbf{n}}^{\sigma}=|\mathbf{n} \sigma\rangle\langle\mathbf{n},-\sigma| .
$$

In Mori's projection operator formalism [13] the following general formula for the imaginary part of the magnetic susceptibility can be derived [15]:

$$
\chi^{\prime \prime}(\mathbf{k} \omega)=-\frac{4 \mu_{B}^{2} \omega \Im R(\mathbf{k} \omega)}{\left[\omega^{2}-\omega f_{\mathbf{k}} \Re R(\mathbf{k} \omega)-\omega_{\mathbf{k}}^{2}\right]^{2}+\left[\omega f_{\mathbf{k}} \Im R(\mathbf{k} \omega)\right]^{2}} .
$$

In Eq. (2), $f_{\mathbf{k}}=\left(i \dot{s}_{\mathbf{k}}^{z},-i \dot{s}_{-\mathbf{k}}^{z}\right)^{-1}$,

$$
(A, B)=i \int_{0}^{\infty} d t\langle[A(t), B]\rangle
$$

with the angular brackets denoting the statistical averaging with the Hamiltonian $H, A(t)=\mathrm{e}^{i H t} A \mathrm{e}^{-i H t}$, $\mu_{B}$ is the Bohr magneton,

$$
s_{\mathbf{k}}^{z}=N^{-1 / 2} \sum_{\mathbf{n}} \mathrm{e}^{i \mathbf{k n}} s_{\mathbf{n}}^{z}
$$

with the number of sites $N, \quad i \dot{s}_{\mathbf{k}}^{z}=\left[s_{\mathbf{k}}^{z}, H\right]$, $\omega_{\mathbf{k}}^{2}=\left(i \dot{s}_{\mathbf{k}}^{z},-i \dot{s}_{-\mathbf{k}}^{z}\right)\left(s_{\mathbf{k}}^{z}, s_{-\mathbf{k}}^{z}\right)^{-1}$,

$$
R(\mathbf{k} \omega)=-i \int_{0}^{\infty} d t \mathrm{e}^{i \omega t}\left(A_{2 t}, A_{2}^{\dagger}\right),
$$

$A_{2}=i^{2} \ddot{s}_{\mathrm{k}}^{z}-\omega_{\mathrm{k}}^{2} s_{\mathrm{k}}^{z} \quad$ with the time dependence $i^{d A_{2 t}} / d t=\left(1-P_{0}\right)\left(1-P_{1}\right)\left[A_{2 t}, H\right], \quad A_{2, t=0}=A_{2}$, and the projection operators $P_{k}, k=0,1$, are defined as $P_{k} Q=\left(Q, A_{k}^{\dagger}\right)\left(A_{k}, A_{k}^{\dagger}\right)^{-1} A_{k}$ where $A_{0}=s_{\mathbf{k}}^{z}$ and $A_{1}=i \dot{s}_{\mathbf{k}}^{z}$. In the normal state for Hamiltonian (1) the parameters in Eq. (2) read [16]

$$
\begin{gathered}
\omega_{\mathbf{k}}^{2}=16 J^{2} \alpha\left|C_{1}\right|\left(1-\gamma_{\mathbf{k}}\right)\left(\Delta+1+\gamma_{\mathbf{k}}\right), \\
f_{\mathbf{k}}^{-1}=4 J\left|C_{1}\right|\left(1-\gamma_{\mathbf{k}}\right), \\
\Im R(\mathbf{k} \omega)=\frac{8 \pi \omega_{\mathbf{k}}^{2}}{N} \times \\
\times \sum_{\mathbf{k}^{\prime}} g_{\mathbf{k k}^{\prime}}^{2} \int_{-\infty}^{\infty} d \omega^{\prime} \frac{n_{F}\left(\omega+\omega^{\prime}\right)-n_{F}\left(\omega^{\prime}\right)}{\omega} \times \\
\times A\left(\mathbf{k}^{\prime} \omega^{\prime}\right) A\left(\mathbf{k}+\mathbf{k}^{\prime}, \omega+\omega^{\prime}\right),
\end{gathered}
$$

where $\alpha \sim 1$ is the vertex correction [17], $J$ and $C_{1}=\left\langle s_{\mathbf{n}}^{+1} s_{\mathbf{n}+\mathbf{a}}^{-1}\right\rangle$ are the exchange parameter and spin correlation for neighbor sites, $\gamma_{\mathbf{k}}=\left[\cos \left(k_{x}\right)+\right.$ $\left.+\cos \left(k_{y}\right)\right] / 2$ the interaction constant $g_{\mathbf{k k}^{\prime}}=t_{\mathbf{k}^{\prime}}-$ $-t_{\mathbf{k}+\mathbf{k}^{\prime}}$ with

$$
t_{\mathbf{k}}=\sum_{\mathbf{n}} \mathrm{e}^{i \mathbf{k}(\mathbf{n}-\mathbf{m})} t_{\mathbf{n m}}, \quad n_{F}(\omega)=\left(\mathrm{e}^{\omega / T}+1\right)^{-1},
$$

$A(\mathbf{k} \omega)$ is the hole spectral functions. The real part of $R(\mathbf{k} \omega)$ can be calculated from its imaginary parts and Kramers - Kronig relations.

This quantity influences the frequency of spin excitations ${ }^{\circ} \mathbf{k}$ only near $\mathbf{Q}$. Therefore it is convenient to incorporate $\omega f_{\mathbf{k}} \Re R(\mathbf{k} \omega)$ in $\omega_{\mathbf{k}}^{2}$. A positive value of the parameter $\Delta$ [see Eq. (3)] leads to a finite frequency of spin excitations at $\mathbf{Q}$ which differentiates them from the classical antiferromagnetic magnons. Using Eq. (3) it can be shown that ${ }^{\circ} \mathbf{Q} \propto \xi^{-1} \propto x^{1 / 2}$ [16] where $\xi$ is the correlation length of the short-range antiferromagnetic order. Thus, the spin gap at $\mathbf{Q}$ grows with the hole concentration and temperature. The dispersion of spin excitations near the antiferromagnetic momentum is shown in Fig. 1.

If the hole hopping to nearest and next nearest sites is taken into account the interaction constant $g_{\mathbf{k k}^{\prime}}$ between holes and spin excitations acquires the form

$$
g_{\mathbf{k k}^{\prime}}=t\left(\gamma_{\mathbf{k}^{\prime}}-\gamma_{\mathbf{k}+\mathbf{k}^{\prime}}\right)+t^{\prime}\left(\gamma^{\prime} \mathbf{k}^{\prime}-\gamma^{\prime} \mathbf{k}+\mathbf{k}^{\prime}\right) \text {, }
$$

where $\gamma^{\prime} \mathbf{k}=\cos \left(k_{x}\right) \cos \left(k_{y}\right)$. The constant vanishes for $\mathbf{k}=\mathbf{Q}$ when the vector $\mathbf{k}^{\prime}$ is located at the boundary of the magnetic Brillouin zone. In other words, fermions near hot spots interact weakly with spin excitations. This is connected with the short-range character of the interaction - the decaying spin excitation on site $\mathbf{n}$ creates the fermion pair on the same and neighboring sites which is reflected in the above form of the interaction constant. 


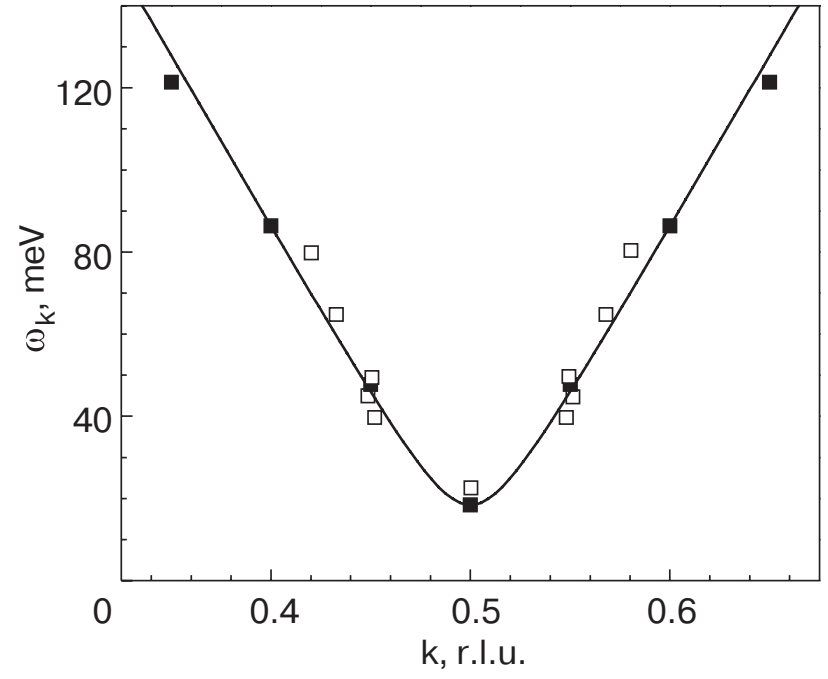

Fig. 1. The dispersion of spin excitations calculated selfconsistently in a $20 \times 20$ lattice for $x=0.06$ and $T=17 \mathrm{~K}$ [15] (filled squares). The solid line is the fit of the dependence ${ }^{\omega_{\mathbf{k}}}=\left[{ }^{2} \mathbf{Q}+c^{2}(\mathbf{k}-\mathbf{Q})^{2}\right]^{1 / 2}$ to these data. Open squares are positions of maxima in the odd susceptibility in $\mathrm{YBa}_{2} \mathrm{Cu}_{3} \mathrm{O}_{6.5}(x \approx 0.075$ [18] $)$ at $T=5 \mathrm{~K}$ [5].

In calculating the susceptibility (2) we used the hole spectral function $A(\mathbf{k} \omega)$ obtained in self-consistent calculations [16] and the one-pole approximation $A(\mathbf{k} \omega)=(\eta / \pi)\left[\left(\omega-\varepsilon_{\mathbf{k}}+\mu\right)^{2}+\eta^{2}\right]^{-1}$ with the hole dispersion $\varepsilon_{\mathbf{k}}$ derived from photoemission data in cuprates $[19,20]$. Here $\eta$ and $\mu$ are the artificial broadening and chemical potential, respectively. The Fermi surface for one of these dispersions [20],

$$
\begin{gathered}
\varepsilon_{\mathbf{k}}=-87.9+554.7 \gamma_{\mathbf{k}}-132.7 \gamma_{\mathbf{k}_{\mathbf{k}}}-13.2 \gamma_{2 \mathbf{k}}+92.45 \times \\
\times\left[\cos \left(2 k_{x}\right) \cos \left(k_{y}\right)+\cos \left(k_{x}\right) \cos \left(2 k_{y}\right)\right]-26.5 \gamma^{\prime}{ }_{2 \mathbf{k}},
\end{gathered}
$$

is shown in Fig. 2. In Eq. (5), the coefficients are in millielectronvolts. This type of the Fermi surface is inherent in moderate doping.

First we consider the case of small transfer frequencies when Eq. (2) reduces to

$$
\chi^{\prime \prime}(\mathbf{k} \omega) \approx-4 \mu_{B^{\omega}}^{2} \omega_{\mathbf{k}}^{-4} \Im R(\mathbf{k} \omega) .
$$

As seen from Eq. (3) and Fig. $1, \omega_{\mathrm{k}}^{-4}$ is a decreasing function of the difference $\mathbf{k}-\mathbf{Q}$ which acts in favor of a commensurate maximum peaked at $\mathbf{Q}$ in the momentum dependence of the susceptibility. However, if $\Im R(\mathbf{k} \omega)$ has a pronounced dip at the antiferromagnetic momentum the commensurate maximum splits into several incommensurate peaks. Indeed, as follows from Eq. (3), states with energies $-(1)<\varepsilon_{\mathbf{k}^{\prime}}-\mu<0$ and $0<\varepsilon_{\mathbf{k}+\mathbf{k}^{\prime}}-\mu<\omega$ make the main contribution to $\Im R(\mathbf{k} \omega)$. For small $\omega$ these states are located near the Fermi surface and for $\mathbf{k}=\mathbf{Q}$ they are

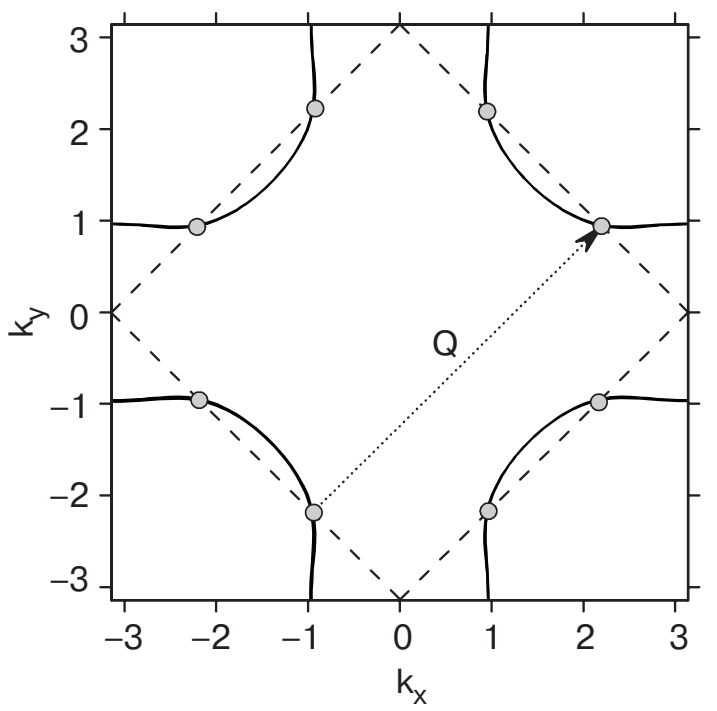

Fig. 2. The Fermi surface for dispersion (5) and $\mu=-40 \mathrm{meV}$ (solid lines). Dashed lines show the boundary of the magnetic Brillouin zone, gray circles are the hot spots, the dotted arrow is the antiferromagnetic momentum $\mathbf{Q}$.

near the hot spots in Fig. 2. As indicated above, for these wave vectors the interaction constant $g_{\mathbf{O k}^{\prime}}$, Eq. (4), is small which leads to the smallness of $\Im R(\mathbf{Q} \omega)$. With the wave vector moving away from $\mathbf{Q}$ momenta of states contributing to the spin-excitation damping recede from the hot spots, the interaction constant grows, and with it the spin-excitation damping. Thus, the damping has a dip at $\mathbf{Q}$ which leads to the low-frequency incommensurability.

The momentum dependence of the normal-state susceptibility corresponding to this latter case is shown in Fig. 3,c. The calculations were carried out using the dispersion (5). Analogous results were also obtained with other dispersions found in literature [19,20]. As seen from the figure, for small frequencies the susceptibility is peaked at the wave vectors $\mathbf{k}=(1 / 2,1 / 2 \pm \delta)$ and $(1 / 2 \pm \delta, 1 / 2)$. Similar momentum dependencies of $\chi^{\prime \prime}(\mathbf{k} \omega)$ were observed in yttrium and lanthanum cuprates $[3,4,9]$.

The dependence of the incommensurability parameter $\delta$ on $x$ calculated in the normal state for $\omega=2 \mathrm{meV}$ is shown in Fig. 4. In agreement with experiment (see the inset) $\delta$ grows with $x$ up to $x \leq 0.12$ and then saturates. In our calculations this dependence is solely conditioned by the behavior of the spin-gap frequency ${ }^{0} \mathbf{Q}$ which for small hole concentrations grows as $x^{1 / 2}$ and saturates near $x=0.12$ [16]. The growth of the spin-gap frequency leads to a weaker momentum dependence of the spin-excitation frequency ${ }^{\circ} \mathbf{k}$ near $\mathbf{Q}$ which increases the distance between susceptibility maxima in Eq. (6). Notice that the change of the hole dispersion which takes place in the concentration 


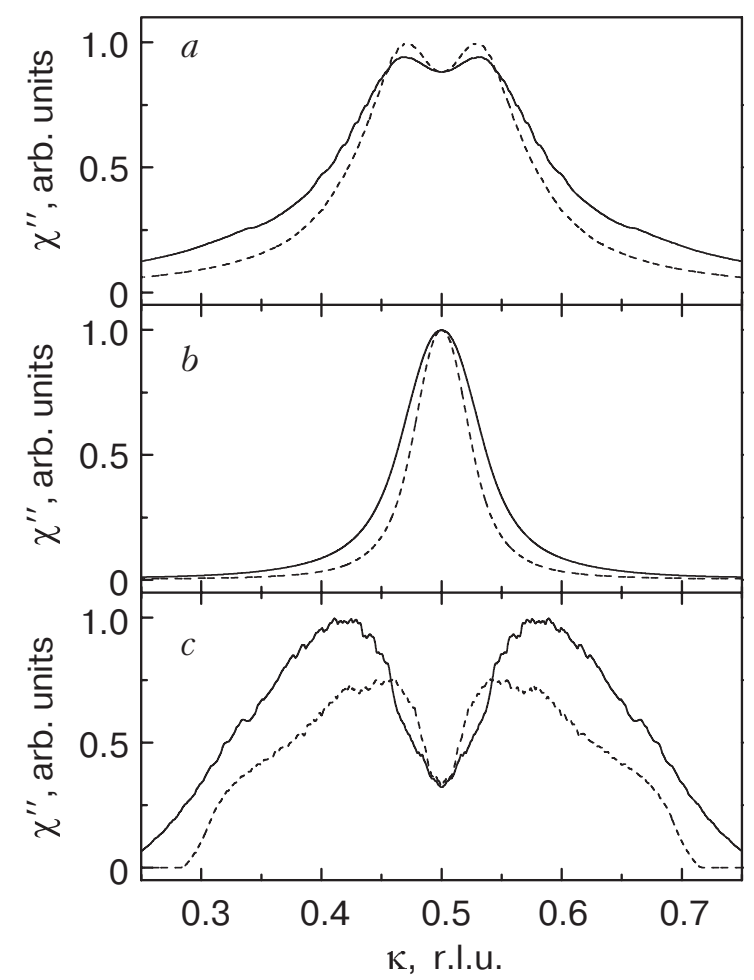

Fig. 3. The momentum dependence of $\chi^{\prime \prime}(\mathbf{k} \omega)$ for $T=0$, $x \approx 0.12, \quad \mu=-40 \mathrm{meV}, \quad t^{\prime}=-0.2 t \quad$ and $\omega=70 \mathrm{meV}$, $\eta=30 \mathrm{meV}(a), \omega=35 \mathrm{meV}, \eta=15 \mathrm{meV}(b), \omega=2 \mathrm{meV}$, $\eta=1.5 \mathrm{meV}(c)$. Calculations were carried out in a $1200 \times 1200$ lattice. The solid lines correspond to scans along the edge of the Brillouin zone, $\mathbf{k}=(\kappa, 1 / 2)$, the dashed lines are for the zone diagonal, $\mathbf{k}=(\kappa, \kappa)$.

range $0.04<x<0.16$ [16] was not taken into account in these calculations.

In the itinerant-electron picture in the normal state the low-frequency incommensurability is related to the Fermi surface nesting [10]. This mechanism imposes rather stringent requirements on the hole energy spectrum, because to reproduce known experimental results the nesting has to persist in a wide doping range with the nesting momentum changed in a specific manner. In the superconducting state the incommensurability stems from the nesting of constant-energy contours $[11,20]$. This mechanism leads to incommensurability also with the use of the more general formula (2) for the susceptibility. However, the mechanism depends crucially on details of the hole dispersion and it is unlikely that it can explain the incommensurability both in lanthanum and yttrium cuprates.

The above discussion was mainly concerned with transfer frequencies $\omega<{ }^{\circ} \mathbf{Q}$. If the frequency approaches ${ }^{{ }} \mathbf{Q}$ the resonance denominator in Eq. (2) starts to dominate in the momentum dependence of the susceptibility. If the spin excitations are not over-

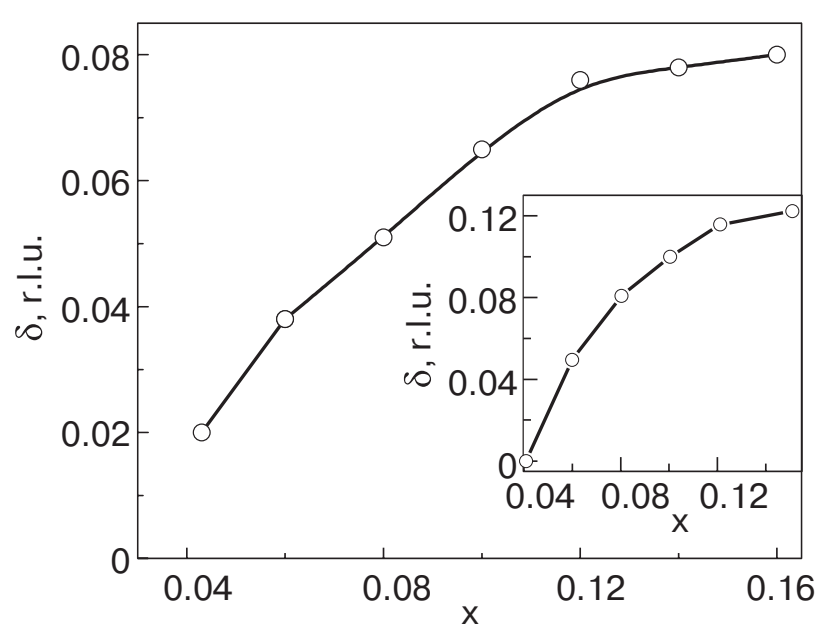

Fig. 4. The incommensurability parameter $\delta$ vs. $x$ for $\omega=2 \mathrm{meV}$. Inset: experimental data [2] for $\mathrm{La}_{2-x} \mathrm{Sr}_{x} \mathrm{CuO}_{4}$. Connecting lines are a guide to the eye.

damped, for $\omega \geq \omega_{\mathbf{Q}}$ the equation $\omega=\omega_{\mathbf{k}}$ defines the position of the maxima in $\chi^{\prime \prime}(\mathrm{k} \omega)$. Using the approximation $\omega_{\mathbf{k}} \approx\left[\omega_{\mathbf{Q}}^{2}+c^{2}(\mathbf{k}-\mathbf{Q})^{2}\right]^{1 / 2}$ which is applicable for $\mathbf{k} \approx \mathbf{Q}$ (see Fig. 1) we find that the maxima are located near a circle centered at $\mathbf{Q}$ with the radius $c^{-1}\left(\omega^{2}-\omega_{\mathbf{Q}}^{2}\right)^{1 / 2}[21,15]$. Thus, for $\omega \approx \omega_{\mathbf{Q}}$ the magnetic response is commensurate, i.e. $\chi^{\prime \prime}(\mathbf{k} \omega)$ is peaked at $\mathbf{Q}$. For larger $\omega$ the commensurate maximum splits into incommensurate peaks with distances from the antiferromagnetic momenta which grow with the frequency. Such situations are shown in Figs. 3, $a$ and $3, b$. For parameters of Fig. $3 \quad \omega_{0} \mathbf{0} \approx 37 \mathrm{meV}$. With these parameters incommensurate maxima for ${ }^{(1)}>{ }^{\circ} \mathbf{Q}$ are located at $\mathbf{k}=(1 / 2 \pm \delta, 1 / 2 \pm \delta)$, $(1 / 2 \pm \delta, 1 / 2 \mp \delta)$, in contrast to the low-frequency maxima at $(1 / 2,1 / 2 \pm \delta),(1 / 2 \pm \delta, 1 / 2)$. A similar location of the high-frequency maxima was observed in $\mathrm{YBa}_{2} \mathrm{Cu}_{3} \mathrm{O}_{7-y}$ and $\mathrm{La}_{2-x} \mathrm{Ba}_{x} \mathrm{CuO}_{4}[4,8,9]$. However, for other parameters, e.g., for increased hole damping, maxima were found to form a circle or merge together in a broad commensurate maximum. Such situations were also observed experimentally [22]. As follows from the above discussion, the dispersion of maxima in $\chi^{\prime \prime}(\mathbf{k} \omega)$ has a shape of two parabolas with upward- and downward-directed branches which converge at the antiferromagnetic momentum $\mathbf{Q}$ and at the frequency ${ }^{\omega} \mathbf{Q}$ which is identified with $\omega_{r}$.

The frequency dependencies of the magnetic susceptibility at the antiferromagnetic momentum for the superconducting and normal states are shown in Figs. 5 and 6 . Our results for the normal state were obtained from the data of the self-consistent calculations [15,16]. For the superconducting state the same ordinary hole self-energies as for the normal state and the $d$-type anomalous self-energy 


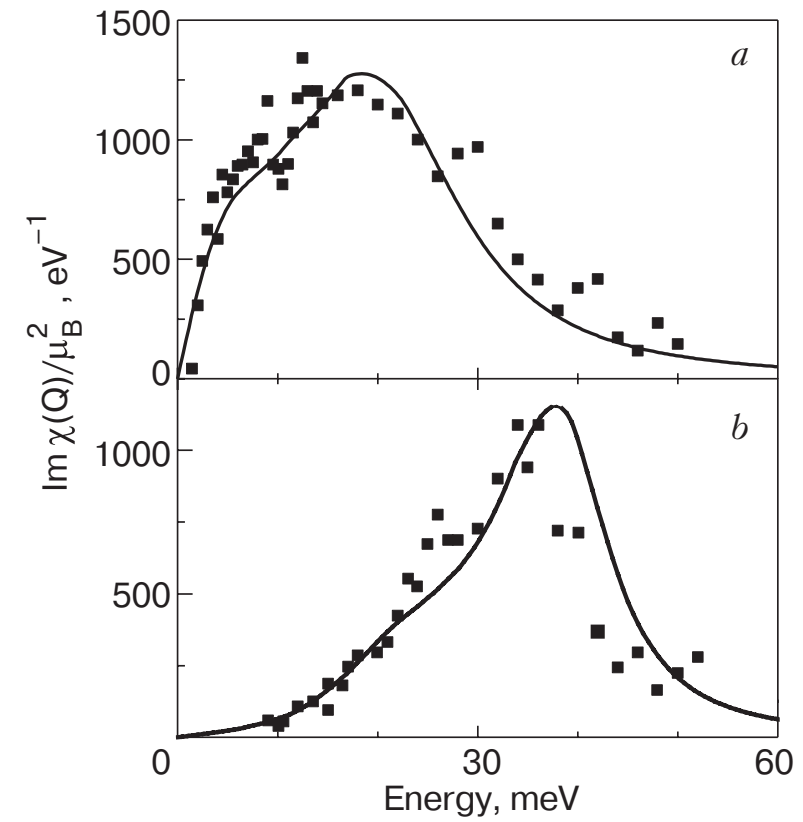

Fig. 5. The frequency dependence of $\chi^{\prime \prime}(\mathbf{Q}())$ in the superconducting state. Curves are our results obtained with the hole spectrum from Ref. 16 for $T=17 \mathrm{~K}, x=0.06(a)$ and $x=0.12(b)$. Filled squares are the odd susceptibility measured [5] in $\mathrm{YBa}_{2} \mathrm{Cu}_{3} \mathrm{O}_{6.5}[(a), x \approx 0.075]$ and in $\mathrm{YBa}_{2} \mathrm{Cu}_{3} \mathrm{O}_{6.83}[(b), x \approx 0.14]$ at $T=5 \mathrm{~K}$.

$\Sigma_{12}(\mathbf{k} \sigma)=\left(\sigma \Delta^{s} / 2\right)\left[\cos \left(k_{x}\right)-\cos \left(k_{y}\right)\right]$ with the superconducting gap $\Delta^{s}=20 \mathrm{meV}$ [23] were used for calculating $\Im R(\mathbf{k} \omega)$. As seen from the figures, the $t-J$ model is able to reproduce correctly the location of the maximum in the susceptibility and gives a proper evolution of this maximum with doping and temperature. For the parameters of Figs. 5 and 6 the location of the maximum coincides approximately with the value of the spin gap ${ }^{\circ} \mathbf{O}$ and is determined by the resonance denominator in Eq. (2). Comparing the spin-excitation damping with the gap frequency we found that the spin excitations are not overdamped near $\mathbf{Q}$. In view of the similarity in shapes of the experimental and calculated curves the same conclusion can be made with respect to the spin excitations in the underdoped $\mathrm{YBa}_{2} \mathrm{Cu}_{3} \mathrm{O}_{7-y}$. Similar results were obtained with the hole dispersion (5).

However, the value of the spin-excitation damping was found to depend heavily on details of the hole band structure such as the bandwidth, damping and the distribution of the spectral weight. Changes in these parameters may lead to overdamping of spin excitations. In this case the position of the maximum in the frequency dependence of $\chi^{\prime \prime}$ has nothing to do with the spin-excitation frequency. An example of such changes is shown in Fig. 7 where the susceptibility was calculated with dispersion (5) scaled by the factor 0.4 [16]. The calculations were carried out for

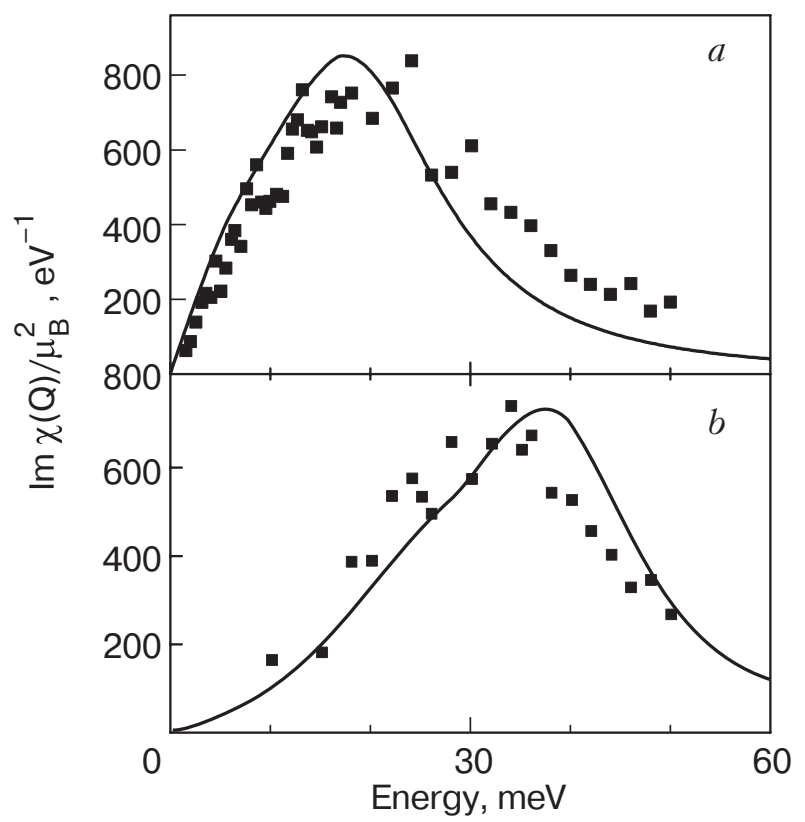

Fig. 6. The frequency dependence of $\chi^{\prime \prime}(\mathbf{Q} \omega)$ in the normal state. Curves are our results obtained with the hole spectrum from Ref. 16 for $T=116 \mathrm{~K}, x=0.06(a)$ and $x=0.12(b)$. Filled squares are the odd susceptibility measured [5] in $\mathrm{YBa}_{2} \mathrm{Cu}_{3} \mathrm{O}_{6.5}(a)$ and $\mathrm{YBa}_{2} \mathrm{Cu}_{3} \mathrm{O}_{6.83}(b)$ at $T=100 \mathrm{~K}$.

$\mathbf{k}=(0.42,0.5)$ which corresponds to the momentum of the low-frequency peak in Fig. 3,c. In this case excitations near the spin gap are overdamped which leads to the red shift of the susceptibility maximum. The similar frequency dependence of $\chi^{\prime \prime}$ without a well-defined peak of spin excitations is observed in $\mathrm{La}_{2-x} \mathrm{Ba}_{x} \mathrm{CuO}_{4}$ [6]. Thus, we suppose that the observed dissimilarity in the frequency dependencies of the susceptibility in lanthanum and yttrium cuprates may be connected with some difference in their hole spectra.

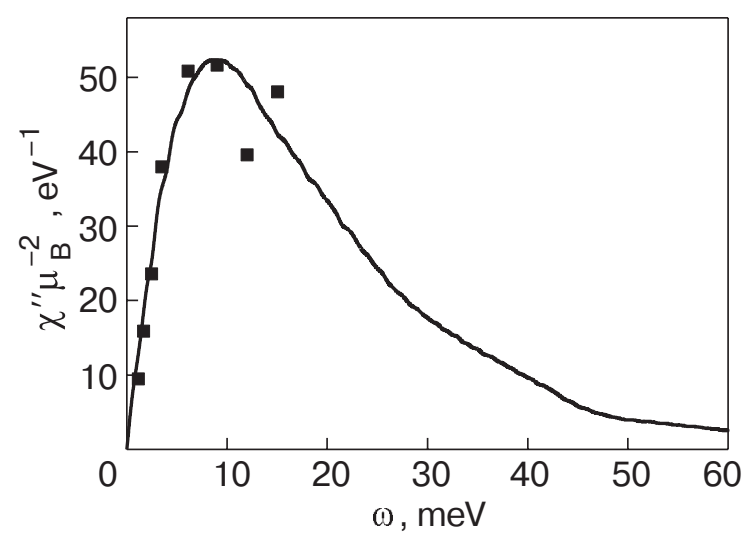

Fig. 7. The frequency dependence of $\chi^{\prime \prime}(\mathbf{Q} \omega)$ in the normal state. The solid curve was calculated for $x \approx 0.12$, $T=0, \mathbf{k}=(0.42,0.5)$ and dispersion (5) scaled by the factor 0.4. Squares are the susceptibility in $\mathrm{La}_{1.86} \mathrm{Sr}_{0.14} \mathrm{CuO}_{4}$ for $T=35 \mathrm{~K}$ at the incommensurate peak [6]. 
In summary, Mori's projection operator formalism and the $t-J$ model of $\mathrm{Cu}-\mathrm{O}$ planes were used for the interpretation of the magnetic susceptibility in cuprate perovskites. It was shown that the calculated momentum and frequency dependencies of the imaginary part of the susceptibility $\chi^{\prime \prime}$, the dispersion and location of maxima in it and the concentration dependence of the incommensurability parameter are similar to those observed in lanthanum and yttrium cuprates. The dispersion of the maxima in $\chi^{\prime \prime}$ resembles two parabolas with upward- and downward-directed branches which converge at the antiferromagnetic wave vector $\mathbf{Q}$ and at the respective frequency of spin excitations ${ }^{\oplus} \mathbf{Q}$. We relate the upper parabola to the spin-excitation dispersion. For the normal state the incommensurability connected with the lower parabola is related to the dip in the spin-excitation damping at $\mathbf{Q}$. For moderate doping the dip arises due to the smallness of the interaction between spin excitations and holes near the hot spots, which is a consequence of the short-range character of this interaction. In agreement with experiment the incommensurate peaks which form the lower parabola are located at momenta $(1 / 2,1 / 2 \pm \delta)$ and $(1 / 2 \pm \delta, 1 / 2)$, while peaks in the upper parabola are at $(1 / 2 \pm \delta, 1 / 2 \pm \delta)$ and $(1 / 2 \pm \delta, 1 / 2 \mp \delta)$. Also in agreement with experiment the low-frequency incommensurability parameter $\delta$ grows with the hole concentration $x$ for $x \leq 0.12$ and then saturates. This behavior of $\delta$ is mainly connected with the concentration dependence of the frequency ${ }^{\circ} \mathbf{Q}$ of the spin gap at the antiferromagnetic wave vector. We suppose that the marked difference in the frequency dependencies of the susceptibility in $\mathrm{YBa}_{2} \mathrm{Cu}_{3} \mathrm{O}_{7-y}$ and $\mathrm{La}_{2-x} \mathrm{Sr}_{x} \mathrm{CuO}_{4}$ is a consequence of the difference in the electron spectra. The larger spin-excitation damping in $\mathrm{La}_{2-x} \mathrm{Sr}_{x} \mathrm{CuO}_{4}$ leads to overdamping of spin excitations, while in the underdoped $\mathrm{YBa}_{2} \mathrm{Cu}_{3} \mathrm{O}_{7-y}$ the excitations are well-defined even in the normal state.

This work was partially supported by the ESF grant No. 5548 and by the DFG.

1. H. Yoshizawa, S. Mitsuda, H. Kitazawa, and K. Katsumata, J. Phys. Soc. Jpn. 57, 3686 (1988).

2. K. Yamada, C.H. Lee, K. Kurahashi, J. Wada, S. Wakimoto, S. Ueki, H. Kimura, Y. Endoh, S. Hosoya, G. Shirane, R.J. Birgeneau, M. Greven, M.A. Kastner, and Y.J. Kim, Phys. Rev. B57, 6165 (1998).
3. T.E. Mason, G. Aeppli, S.M. Hayden, A.P. Ramirez, and H.A. Mook, Phys. Rev. Lett. 71, 919 (1993).

4. P. Dai, H.A. Mook, and F. Dogan, Phys. Rev. Lett. 80, 1738 (1998).

5. P. Bourges, in: The Gap Symmetry and Fluctuations in High Temperature Superconductors, J. Bok, G. Deutscher, D. Pavuna, and S.A. Wolf (eds.), Plenum Press, New York (1998), p. 349.

6. G. Aeppli, T.E. Mason, S.M. Hayden, H.A. Mook, and J. Kulda, Science 279, 1432 (1997).

7. S.M. Haiden, G. Aeppli, H.A. Mook, T.G. Perring, T.E. Mason, S.-W. Cheong, and Z. Fisk, Phys. Rev. Lett. 76, 1344 (1996).

8. S.M. Haiden, H.A. Mook, P. Dai, T.G. Perring, and F. Dogan, Nature 429, 531 (2004).

9. J.M. Tranquada, H. Woo, T.G. Perring, H. Goka, G.D. Gu, G. Xu, M. Fujita, and K. Yamada, Nature 429, 534 (2004).

10. D.Z. Liu, Y. Zha, and K. Levin, Phys. Rev. Lett. 75, 4130 (1995).

11. J. Brinckmann and P.A. Lee, Phys. Rev. Lett. 82, 2915 (1999).

12. V. Hizhnyakov and E. Sigmund, Physica C156, 655 (1988); J. Zaanen and O. Gunnarsson, Phys. Rev. B40, R7391 (1989).

13. H. Mori, Progr. Theor. Phys. 34, 399 (1965).

14. Yu.A. Izyumov, Usp. Fiz. Nauk 167, 465 (1997) [Phys.-Usp. (Russia) 40, 445 (1997)].

15. A. Sherman and M. Schreiber, Phys. Rev. B65, 134520 (2002); ibid. B68, 094519 (2003).

16. A. Sherman and M. Schreiber, Eur. Phys. J. B32, 203 (2003); A. Sherman, Phys. Rev. B70, 184512 (2004).

17. J. Kondo and K. Jamaji, Progr. Theor. Phys. 47, 807 (1972).

18. J.L. Tallon, C. Bernhard, H. Shaked, R.L. Hitterman, and J.D. Jorgensen, Phys. Rev. B51, R12911 (1995).

19. M.C. Schabel, C.-H. Park, A. Matsuura, Z.-X. Shen, D.A. Bonn, R. Liang, and W.N. Hardy, Phys. Rev. B57, 6090 (1998).

20. M.R. Norman, Phys. Rev. B61, 14751 (2000).

21. V. Barzykin and D. Pines, Phys. Rev. B52, 13585 (1995)

22. A. Stock, W.J.L. Buyers, R.A. Cowley, P.S. Clegg, R. Coldea, C.D. Frost, R. Liang, D. Peets, D. Bonn, W.N. Hardy, and R.J. Birgeneau, Phys. Rev. B71, $024522(2005)$

23. N.-C. Yeh, C.-T. Chen, R.P. Vasquez, C.U. Jung, S.-I. Lee, K. Yoshida, and S. Tajima, J. Low Temp. Phys. 131, 435 (2003). 